\title{
The Impact of Thermal Modeling on Limiting RF-EMF*
}

\author{
Norbert Leitgeb \\ Institute of Health Care Engineering with European Notified Body of Medical Devices, Graz University of Technology, Graz, Aus- \\ tria. \\ Email: norbert.leitgeb@tugraz.at
}

Received February $5^{\text {th }}, 2013$; revised March $8^{\text {th }}, 2013$; accepted March $21^{\text {st }}, 2013$

Copyright (C) 2013 Norbert Leitgeb. This is an open access article distributed under the Creative Commons Attribution License, which permits unrestricted use, distribution, and reproduction in any medium, provided the original work is properly cited.

\begin{abstract}
The paper quantitatively assesses the relationship between specific energy absorption rates $(S A R)$ of radio frequency (RF) electromagnetic fields (EMF) and resulting intracorporal tissue temperature changes $(\Delta T)$ at whole body exposure of a small person to resonant RF EMF. Applied thermal modeling allowed accounting also for dynamic thermoregulatory responses. As expected from physical laws the correlation of all local $S A R$ values and $\triangle T$ data was fairly good. However, at local level $S A R$ proved to be only weakly associated with $\triangle T$. Even if averaged, over any $10 \mathrm{~g}$ tissue the ratio $\Delta T_{10 \mathrm{~g}} / S A R_{10 \mathrm{~g}}$ still varied by almost two orders of magnitudes. Blood perfusion was found to play a major role in affecting local temperature changes and caused even net cooling. The results demonstrate that local $S A R$ is a poor surrogate for local temperature change, and that conventional static thermal modeling underestimates body core temperature. Results demonstrated that recommended reference levels of RF EMF fail to reliably prevent from exceeding yet legally binding basic restrictions not only with regard to whole-body $S A R$ but also with regard to whole-body temperature rise $(\Delta T)$. Consequently, from a legal point of view general presumption of conformity is no longer justified. While thermal basic restrictions were exceeded if related to the whole-body averaged value, compliance could not be excluded with regard to body-core related values. Further results might allow improving EMF limiting in terms of relating it more closely to the basic health-relevant parameter which is tissue temperature change.
\end{abstract}

Keywords: Electromagnetic Fields; Radio Frequency; Health Risks; Tissue Heating; Specific Absorption Rate

\section{Introduction}

Existing regulations and guidelines for limiting exposures to non-ionizing electromagnetic fields (EMF) such as from the International Commission on Non-Ionizing Radiation Protection (ICNIRP) or the Institute of Electrical and Electronics Engineers (IEEE) aim at defining basic limits for those induced intracorporal quantities which primarily generate health-relevant biological effects. For pragmatic reasons in a second step reference values are derived by numerical modeling linking measurable external field parameters to intracorporal basic quantities [1-4].

For biophysical reasons the frequency range of nonionizing EMF (up to $300 \mathrm{GHz}$ ) can be divided into two basic sections governed by quite different but well established interaction mechanisms.

In the extremely low frequency (ELF) range electric and magnetic fields have to be assessed separately although both induce intracorporal electric field strengths

\footnotetext{
*The author declares no conflict of interest.
}

(and in turn electric current densities), however, with different pathways. Induced electric field strengths may cause the primary biologic effect, namely cellular excitation, provided stimulation thresholds are exceeded. Progress in numerical simulation and digital anatomical modeling allowed more reliably assessing the relationship between magnetic reference levels and basic restrictions which in the meanwhile resulted in doubling former magnetic field reference levels (e.g. at power frequency from $100 \mu \mathrm{T}$ to $200 \mu \mathrm{T}$ ) without challenging the basic restriction [1].

In the radio frequency (RF) range, the situation is somewhat more difficult. While there is widespread consensus that the basic interaction mechanism is tissue heating by absorbed RF EMF energy, until recently the obvious basic biologic quantity, namely induced intracorporal tissue temperature change $(\Delta T)$, could not be evaluated with sufficient accuracy, and consequently not reliably linked to external EMF parameters. Therefore, so far, the intracorporal specific rate of RF EMF energy absorption $(S A R)$ is chosen as surrogate for the primary 
parameter $\Delta T$ that is responsible for established biologic effects $[5,6]$. In the meanwhile, progress in numerical simulation and digital anatomical modeling allowed demonstrating that RF EMF reference values are not conservative enough to justify presumption of conformity with basic restrictions in any case. Studies reported up to $45 \%$ excess of basic restrictions at exposure scenarios of persons with small body height such as children or undersized adults [7-15].

Limiting EMF exposure by basic restrictions and reference levels is based on three essential requirements:

a) knowledge of the primary health-relevant interaction mechanisms for identifying basic intracorporal quantities;

b) knowledge of the frequency-dependent dose response as an indispensable requirement for extrapolation to other exposure conditions and limit setting in the entire frequency range;

c) knowledge of the relationship between basic intracorporal quantities and measurable external field parameters (reference levels).

This paper quantitatively assesses the relationship of EMF-induced intracorporal tissue temperature changes and $S A R$ with particular consideration of dynamic thermoregulation.

\section{Method}

Numerical investigations were made in a two-step approach. In a first step, the intracorporal SAR distribution has been calculated by the commercial software package CST Studio Suite [16] based on finite integration technique (FIT) [17] at the numerical anatomical model DIZZY of an 8-year-old boy $(1.4 \mathrm{~m}, 26 \mathrm{~kg})$ segmented into $2 \times 2 \times 2 \mathrm{~mm}$ voxels [18]. In a second step the calculated SAR distributions were used for thermal simulation which was based on the bioheat equation [19] which had been further improved by accounting for additional and time-dependent thermoregulatory processes. These were heat-dependent changing metabolic rate $A$, blood perfusion and vasodilatation $B$, heat exchange $S$ via tissue/air interfaces at the skin and within the lung, and by heat transfer due to insensible water loss and sweating [7]. This resulted in the following improved bioheat equation

$$
\begin{aligned}
& \frac{\partial T(\boldsymbol{r}, \boldsymbol{t})}{\partial t}=\frac{1}{\rho(\boldsymbol{r}) C(\boldsymbol{r})} \\
& \times[(\nabla K(\boldsymbol{r}) \nabla T(\boldsymbol{r}, t))+A(\boldsymbol{r}, T)-S(\boldsymbol{r}, T) \\
& \left.\quad-B(\boldsymbol{r}, T)\left(T(\boldsymbol{r}, t)-T_{B}(t)\right)+\rho(\boldsymbol{r}) \cdot S A R(\boldsymbol{r}, t)\right]
\end{aligned}
$$

with the spatial vector $r$ in $\mathrm{m}$, the time $t$ in $\mathrm{s}$, the specific heat capacity $C$ in $\mathrm{Ws} /(\mathrm{K} \cdot \mathrm{kg})$, the mass density $\rho$ in $\mathrm{kg} / \mathrm{m}^{3}$, the thermal conductivity $K$ in $\mathrm{W} /(\mathrm{m} \cdot \mathrm{K})$, the metabolic rate $A$ in $\mathrm{W} / \mathrm{m}^{3}$, the heat loss at tissue/air interface voxels $S$ in $\mathrm{W} / \mathrm{m}^{3}$, the blood flow coefficient $B$ in $\mathrm{W} /\left(\mathrm{K} \cdot \mathrm{m}^{3}\right)$, and the absorbed energy rate $S A R$ in $\mathrm{W} / \mathrm{kg}$.

After initially calculating the steady-state starting condition, induced tissue temperature changes were determined as a function of time over $60 \mathrm{~min}$ to assure $\Delta T$ reached the new steady-state. Dielectric tissue parameters were derived from the Cole-Cole model [20-22], thermal parameters were taken from the literature [23,24].

The association between $S A R$ and tissue temperature change $\Delta T$ has been quantitatively investigated for a worst-case scenario with plane-wave whole-body RF EMF exposure at resonance frequency and at reference level as defined by exposure limits [2,3].

\section{Results}

The resonance frequency of the used $1.4 \mathrm{~m}$ tall child model at plane wave RF EMF exposure was defined by peaking whole-body $S A R\left(S A R_{W B}\right)$. It was found at 90 $\mathrm{MHz}$. The results showed that reference level RF EMF induce an $S A R_{W B}$ of $0.098 \mathrm{~W} / \mathrm{kg}$ which exceeds the basic restriction $0.08 \mathrm{~W} / \mathrm{kg}$ by $23 \%$. Thermal modeling demonstrated that the temperature increase $\Delta T_{W B}$, averaged over the entire body (including extremities outside the temperature equilibration body-core zone) is only $0.031 \mathrm{~K}$ (Table 1). It has to be noted that hot-spots encountered at the extremities make the whole-body averaged temperature increase $\Delta T_{W B}$ by $61 \%$ larger than the body-core temperature increase $\Delta T_{B C}$ which is averaged over head and trunk only. Consequently, the blood temperature increase which is equivalent to the change of body core temperature $\Delta T_{B C \text { - }}$ amounted to only $0.022^{\circ} \mathrm{K}$ which is $27 \%$ lower than $\Delta T_{W B}$.

If $S A R$ would be used as a surrogate for temperature increase, the relationship of both quantities $S A R$ and $\triangle T$ would be needed to be known. Based on whole-body averages the ratio $\Delta T_{W B} / S A R_{W B}$ was $0.31 \mathrm{~K} /(\mathrm{W} / \mathrm{kg})$ while $\Delta T_{B C} / S A R_{B C}$ was $0.37 \mathrm{~K} /(\mathrm{W} / \mathrm{kg})$. To investigate the variability of the relationship between local $\Delta T$ and $S A R$, vertical profiles of slice-averages of horizontal cross sections $S A R_{\text {slice }}$ and related temperature changes $\Delta T_{\text {slice }}$ were determined. Figure 1 shows that both vertical

Table 1. Characteristic values of RF-EMF induced heating in the DIZZY child model.

\begin{tabular}{cccc}
\hline \multirow{2}{*}{$\begin{array}{c}\text { Region of } \\
\text { interest }\end{array}$} & $S A R_{a v}$ & $\Delta T_{a v}$ & $\Delta T_{a v} / S A R_{a v}$ \\
\cline { 2 - 4 } & $\mathrm{W} / \mathrm{kg}$ & $\mathrm{K}$ & $\mathrm{K} /(\mathrm{W} / \mathrm{kg})$ \\
\hline Whole body & 0.098 & 0.031 & 0.31 \\
Head \& trunk & 0.061 & 0.022 & 0.37 \\
Extremities & 0.159 & 0.044 & 0.27 \\
\hline
\end{tabular}



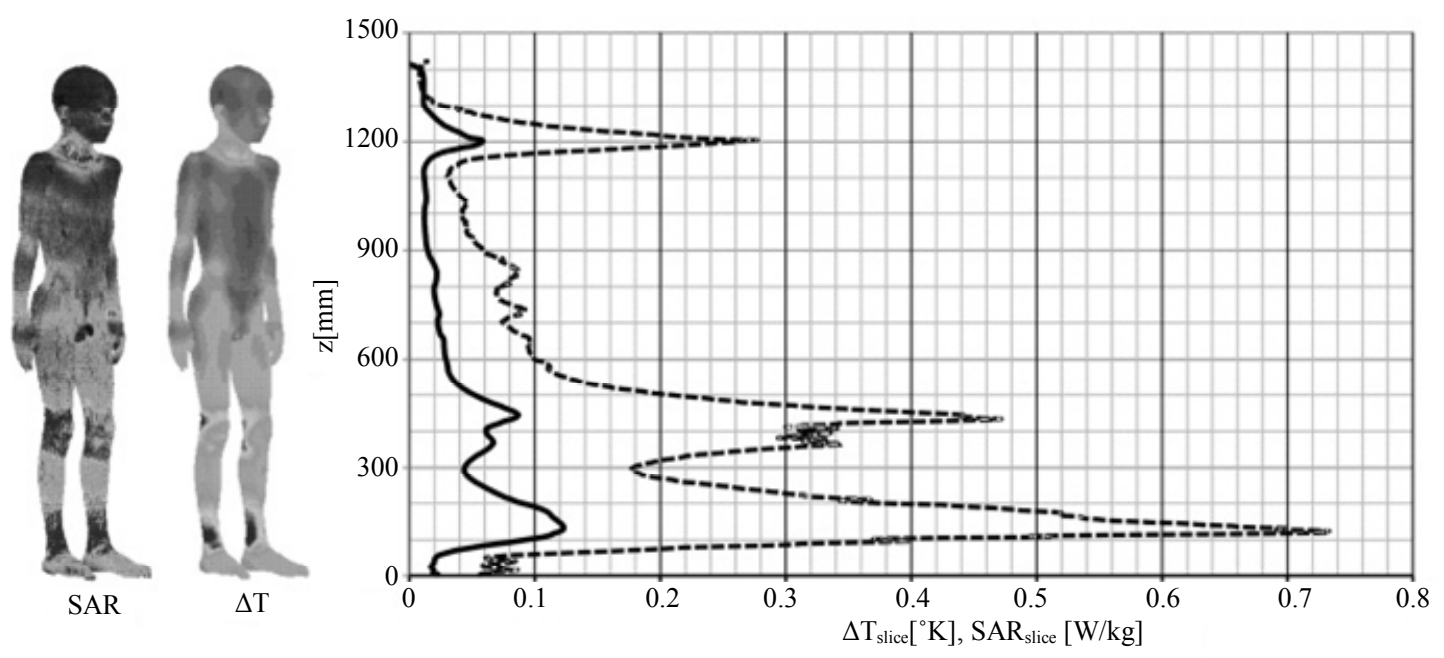

Figure 1. Height profiles of slice-averaged values of $S A R_{\text {slice }}$ and $\Delta T_{\text {slice }}$ with surface distributions of $S A R$ and $\Delta T$ at the child model.

profiles are fairly similar. However, while their overall correlation was very good (correlation coefficient 0.987 ) the ratio $\Delta T_{\text {slice }} / S A R_{\text {slice }}$ varied considerably, in particular up to 6.7-fold in the head region and up to 2.3fold in the remaining body region, respectively. The ratio peaked at the skull because of weak local absorption (low $\left.S A R_{\text {voxel }}\right)$ and heat transfer from the cerebrospinal fluid and brain regions causing an over-proportional temperature increase. The overall span of the ratio

$\Delta T_{\text {slice }} / S A R_{\text {slice }}$ amounted to almost one order of magnitude $(0.177$ to 1.486$)$.

At voxel level, the association between $S A R_{\text {voxel }}$ and $\Delta T_{\text {voxel }}$ was weaker than at slice-averaged values, but still fairly good (correlation coefficient 0.808). However, detailed analysis of the $3.1 \times 10^{6}$ voxel-values showed that local variations of $S A R_{\text {voxel }}$ and $\Delta T_{\text {voxel }}$ values are rather high. The maximum voxel-values were $4.03 \mathrm{~W} / \mathrm{kg}$ and $0.24^{\circ} \mathrm{K}$, respectively. Interestingly, if drawn one over the other the distribution exhibits a complex structure indicating that the relationship between $S A R_{\text {voxel }}$ and $\Delta T_{\text {voxel }}$ is governed by physiologic heat transfer mechanisms. To demonstrate this, results are presented one over the other (in both versions) for the extremities and the temperature-regulated body core (head and trunk). Figure 2 shows that the temperature elevation within the temperature-controlled body-core region is clearly lower than at the extremities. In addition, since physiological heat transfer tends to decrease $\Delta T_{\text {voxel }}$ in voxels with higher absorption rate (heat sources) and to increase $\Delta T_{\text {voxel }}$ at voxels with lower $S A R$ (heat sinks) the distribution exhibits a two-tear structure which is clearly seen in the head and trunk (torso) data (Figure 2). At local level enforced heat dissipation by increased blood circulation can even lead to negative heat balance and negative values of $\Delta T_{\text {voxel }}$ (net cooling). Please note that the chosen logarithmic presentation does not allow including negative values; these were omitted.

The role of blood perfusion was investigated by analyzing differently perfused tissues such as muscle and fat. Figure 3 shows that due to its higher water content and better perfusion muscle exhibits higher $S A R_{\text {voxel }}$ but smaller $\Delta T$. Almost the same temperature elevations were found in fat tissue at lower $S A R_{\text {voxel }}$. Due to lower heat dissipation, the same amount of absorbed energy induced higher temperature elevations in fat compared to muscle tissue. Consequently, overall, the distribution associated with fat tissue is shifted towards lower values of $S A R_{\text {voxel }}$ (Figure 3).

If $S A R$ was a good proxy for $\Delta T$, the ratio of both quantities should be fairly constant. However, this is clearly not the case. This is reflected already by the ratio of medians $\triangle T / S A R$ of the entire fat and muscle tissue. The ratio was 1.85 -fold higher at fat compared to muscle tissue with $0.5 \mathrm{~K} /(\mathrm{W} / \mathrm{kg})$ and $0.27 \mathrm{~K} /(\mathrm{W} / \mathrm{kg})$, respectively.

The ratio of voxel-values $\Delta T_{\text {voxel }} / S A R_{\text {voxel }}$ varied even more. Ideally, it should be independent on SAR. In fact, it is not. It is systematically decreasing with increasing $S A R_{\text {voxel }}$ (Figure 4). The overall span is extremely large and amounts to about 4 orders of magnitudes. Evaluated over the whole body, the ratio may exhibit even negative values (which for logarithmic presentation are cut off in Figure 4). The ratio

$\Delta T_{\text {voxel }} / S A R_{\text {voxel }}$ ranges from $-20 \mathrm{~K} /(\mathrm{W} / \mathrm{kg})$ to at most $45 \mathrm{~K} /(\mathrm{W} / \mathrm{kg})$ which is 145 -fold higher than the ratio $0.31 \mathrm{~K} /(\mathrm{W} / \mathrm{kg})$ of whole-body averaged values.

Since recommended basic restrictions of RF EMF exposure are based on spatial averages over any $10 \mathrm{~g}$ tissue $\left(S A R_{10 \mathrm{~g}}\right)$, the distribution of $S A R_{10 \mathrm{~g}}$ has also been analyzed. Compared to $S A R_{\text {voxel }}$ the correlation of all values 

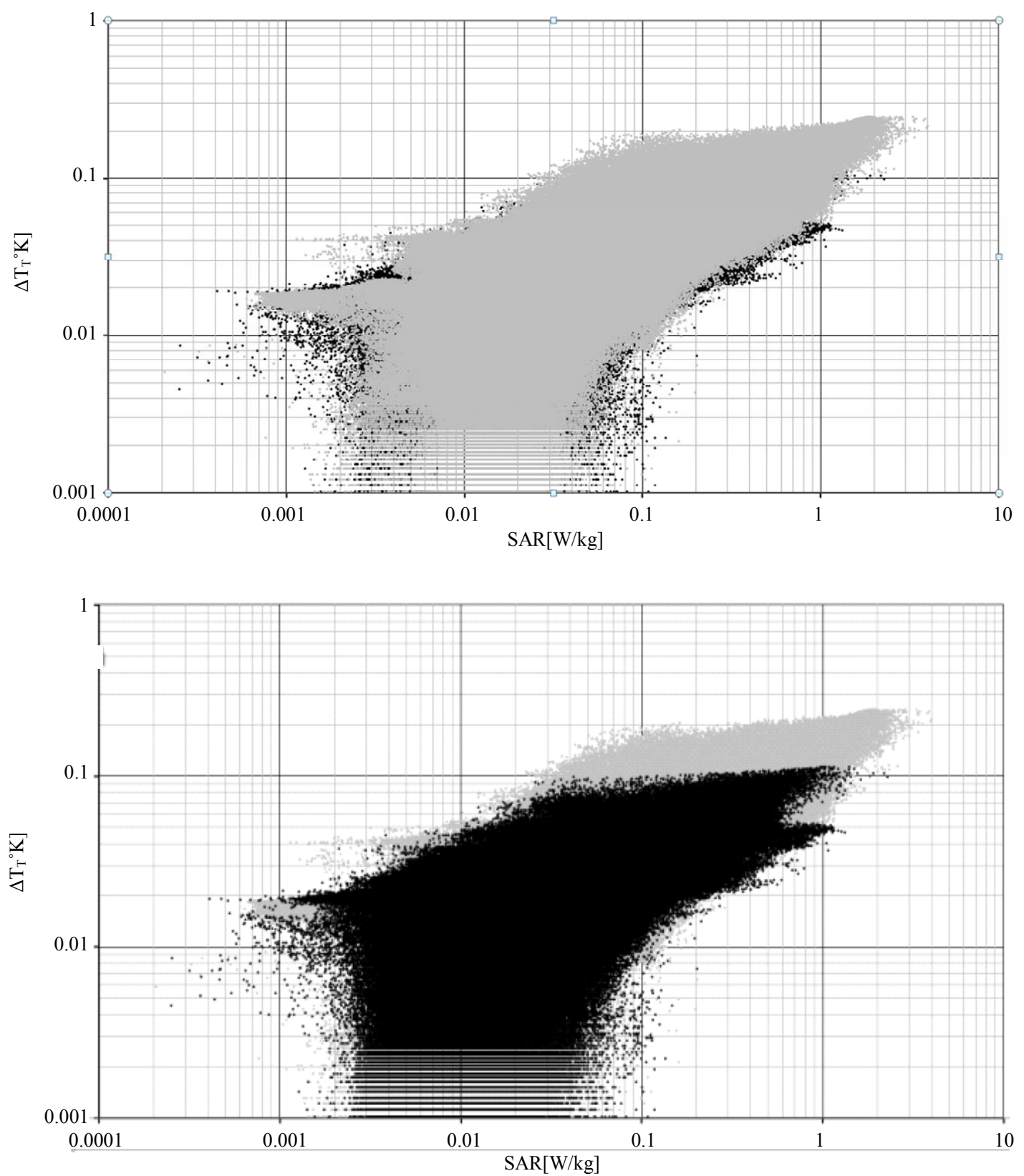

Figure 2. Region-specific voxel-values $\Delta T$ over $S A R$ at the child model; extremities over torso (above) and torso over extremities (below); torso (black), extremities (grey).

$S A R_{10 \mathrm{~g}}$ and $\Delta T_{10 \mathrm{~g}}$ improved to 0.977 , and variability became smaller. The maxima of $S A R_{10 \mathrm{~g}}$ and $\Delta T_{10 \mathrm{~g}}$ were $1.19 \mathrm{~W} / \mathrm{kg}$ and $0.186^{\circ} \mathrm{C}$, respectively. Due to averaging, no (local) negative values of temperature changes were found anymore. However, within the body the ratio $\Delta T_{10 \mathrm{~g}} / S A R_{10 \mathrm{~g}}$ still varied considerably. It exhibited a span of almost two orders of magnitudes reaching from $0.11 \mathrm{~K} /(\mathrm{W} / \mathrm{kg})$ up to a maximum of $9.63 \mathrm{~K} /(\mathrm{W} / \mathrm{kg})$. The maximum $\Delta T_{10 \mathrm{~g}} / S A R_{10 \mathrm{~g}}$ was still 38.3 -fold higher than the ratio of whole-body averages $\Delta T_{W B} / S A R_{W B}$ while the minimum was 2.4 -fold lower.

\section{Discussion}

Any modeling is inherently suffering from unavoidable simplifications and numerical restrictions. This leads to deviations from the real world, and consequently to uncertainties. Sources of uncertainty are discretization, meshing, computational space limitation, boundary conditions, physical tissue properties, and computational algorithms, both for EMF interaction, heating and thermal regulation. To minimize uncertainties, a high spatial resolution of $2 \mathrm{~mm}$ was chosen, which among others allowed minimizing staircase effects; meshing was made 

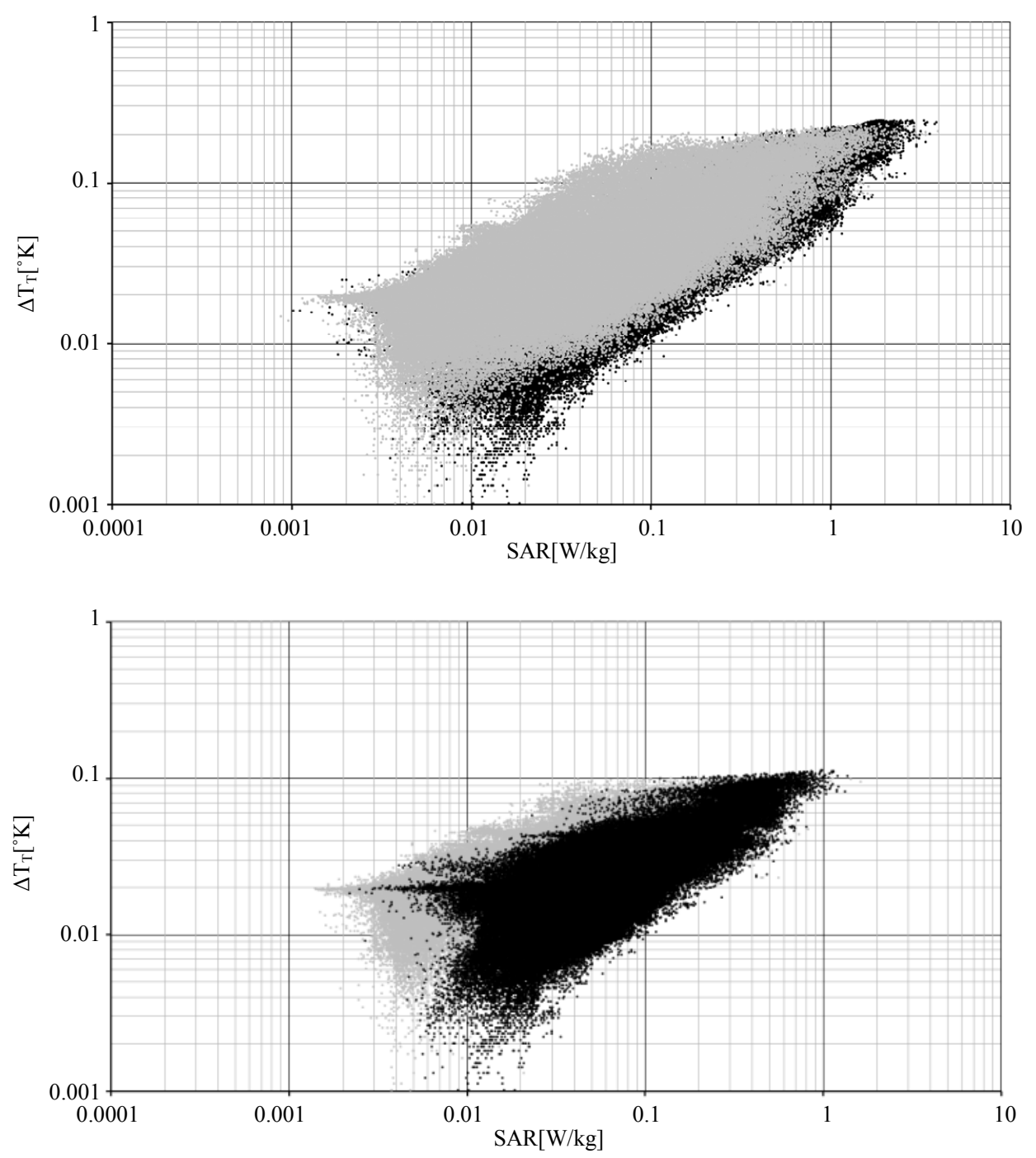

Figure 3. Tissue-specific voxel-values $\Delta T$ over $S A R$ at the head and trunk region of the child model, fat over muscle (above) and muscle over fat(below); muscle (black), fat tissue (grey).

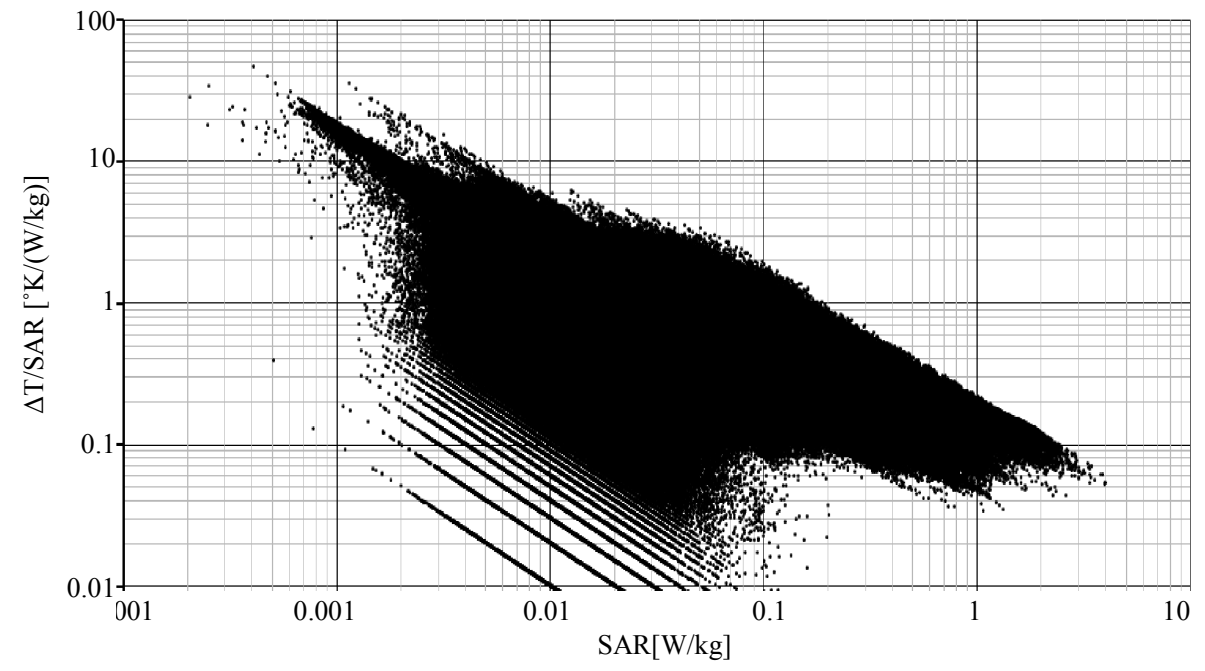

Figure 4. Voxel-related ratio $\triangle T / S A R \quad$ over $S A R$ at the child model. 
such as to avoid compromising the high spatial resolution, boundaries were non-reflective and isothermal. This allowed limiting uncertainty to about $1 \%$ in accordance to [25]. The uncertainty with regard to tissue properties is caused by their measurement itself, extrapolation from animal tissue to humans and interpersonal variability. The related uncertainty can be estimated to about $10 \%$ $15 \%$ for physical and about $10 \%$ for thermal parameters, respectively [20-22]. The uncertainty of the numerical algorithm was minimized by carefully setting the termination criteria and can be estimated to less than 5\% [16].

The overall performance of the numerical SAR estimation was validated by using the test model as defined in the standard EN 62311 [26] which resulted in a deviation of EMF-induced quantities of $2.8 \%$. Thermal modeling was validated by simulating experiments with provoked body heating caused by exposure to $450 \mathrm{MHz}$ EMF [27]. The deviation from experimental results remained below $20 \%$. This includes overall uncertainties of numerical modeling and those of the experimental approach [28].

In the rational of recommended guidelines the objective of EMF limitation is declared to prevent from excess heating, in particular from whole-body heating exceeding $1{ }^{\circ} \mathrm{C}[1,3]$. The presented results with improved thermal modeling shows, that rather than whole-body-heating the body core temperature rise $\Delta T_{B C}$ of $0.022^{\circ} \mathrm{C}$ is almost low enough to allow concluding that this goal is met incases of whole body exposure to reference values even in excess of present $S A R_{W B}$ limits. The low temperature elevation is not surprising since in case of induced heat, thermoregulation aims at keeping body's core temperature constant. However, this does not exclude higher temperature riseat local level. Based on the initial bioheat equation, Bakker et al. [8] reported a maximum local temperature rise of $1^{\circ} \mathrm{C}$. By accounting for dynamic thermoregulatory mechanisms in our study the maximum voxel temperature rise is somewhat lower with a $\Delta T_{\text {voxel }}$ of $0.24^{\circ} \mathrm{C}$. It should be noted that locally averaged values are generally lower than voxel-values. The $\Delta T_{10 \mathrm{~g}}$ value which was encountered at the extremities was at most $0.18^{\circ} \mathrm{C}$.

One particular finding of this study was that good correlation of the entire voxel data of $\triangle T_{\text {voxel }}$ and $S A R_{\text {voxel }}$ is not sufficient to take $S A R$ as a surrogate for temperature increase. In fact this does not already indicate that local $S A R$ was a good proxy, neither for $\Delta T$ at voxel level nor at $10 \mathrm{~g}$ average level. The reason is that of course good correlation of the entire data is not surprising and follows already from the bioheat equation. However, biological thermoregulation and tissue-specific heating cause the large variance in the relationship between $S A R$ and $\Delta T$ at local level which increases with decreasing averaging volume down to voxels. However, in spite of their fairly good overall correlation even averages over any $10 \mathrm{~g}$ tissue still exhibit considerable variance. These results challenge conclusions drawn from previous thermal modeling suggesting that averaging $S A R$ over a volume such as $10 \mathrm{~cm}^{3}(10 \mathrm{~g})$ would be a better predictor of tissue temperature rise although statistically not significantly correlated with $\Delta T_{10 \mathrm{~g}}$ tissue [30,31].

The presented results demonstrate that local temperature rise is strongly dependent on the intensity and dynamics of tissue perfusion. Since body-core temperature is regulated by thermal control mechanisms such as vasodilation and insensible water loss, SAR-attributed temperature changes are smaller in body core regions compared to extremities.

Taking local $S A R$ as proxy for local $\Delta T$ is conservatively ignoring any heat dissipation and thermoregulation and, hence, implicitly assuming adiabatic heating. However, it does not account for heat dissipation to low SARregions which could heat sensible regions even higher than expected. Therefore, if reduction factors were not conservatively chosen, this approach is not necessarily conservative in any case.

The results confirmed that RF EMF reference levels do not prevent small people from exposures exceeding the basic restriction. In this particular case the excess was $23 \%$. This finding is in agreement with the literature reporting overexposures up to $45 \%$ [7-15].

In its statement to its guidelines for limiting exposure to RF EMF ICNIRP acknowledged such results and argued, the excess of recommended basic restrictions would be negligible compared to the large reduction factor 50 chosen for the general public [29]. However, while such an argument is acceptable in scientific discussions on health risks, it is inadequate from a legal point of view, since legal restrictions need to be met anyway and do not leave room for further negotiation.

Therefore, such a non-compliant relationship challenges the presumption of conformity with basic restrictions when meeting reference levels. In our case the excess of basic $S A R$ restrictions also leads to exceeding the implicit basic thermal restriction $0.02^{\circ} \mathrm{C}$ as derived from the objective to prevent from heating above $1^{\circ} \mathrm{C}$ by applying the reduction factor 50 . The excess of the temperature limit was $52 \%$ with regard to the whole-body average and $12 \%$ with regard to the body-core temperature, respectively. It needs to be noted that the latter remains within the uncertainty range and would not already proof noncompliance.

It has to be stressed that calculation with the initial bioheat equation [19] revealed that the thermal basic restriction was exceeded by only $20 \%$ by $\Delta T_{W B}$ while $\Delta T_{B C}$ remained in compliance with it. The reason for this is that the bioheat equation does not allow dynamic changes of the blood temperature. Consequently, constant blood temperature overestimates cooling and subsequently re- 
sults in lower body-core temperatures.

The encountered non-compliance between reference values and basic restrictions requires either adapting reference values and/or basic $S A R_{W B}$ restrictions.

The presented study demonstrates that thermal modeling allows gaining better insight into the relationship between $S A R$ and the objectives of health protection. A potential change from $S A R$ to the biological more relevant quantity $\Delta T$ would require further studies on the impact of worst-case scenarios with regard to environment, biology and interpersonal variance.

\section{Conclusions}

Thermal modeling performed on a small person (child) exposed to resonance frequency $(90 \mathrm{MHz})$ EMF by accounting for time-dependent thermoregulatory processes showed, that in spite of the fairly good correlation between overall data of $S A R_{10 \mathrm{~g}}$ and $\Delta T_{10 \mathrm{~g}}$, local $S A R_{10 \mathrm{~g}}$ values are only weakly associated with local tissue temperature changes. Therefore, local $S A R_{10 \mathrm{~g}}$ is no adequate surrogate to quantitatively assess local tissue temperature changes.

The fact that reference levels do not justify assumption of conformity in any exposure scenario poses a legal problem and requires a solution.

The results confirmed the reported excess of wholebody basic $S A R_{W B}$ restrictions at plane wave EMF exposure of undersized people at reference levels. They demonstrated also that also deduced thermal basic restrictions might be exceeded if related to the whole-body average. However, compliance could not be excluded with regard to body-core averaged temperature rise.

\section{REFERENCES}

[1] ICNIRP, "Guidelines for Limiting Exposure to TimeVarying Electric and Magnetic Fields (1 Hz to $100 \mathrm{kHz}$ )," Health Physics, Vol. 99, No. 6, 2010, pp. 818-836.

[2] ICNIRP, "Guidelines for Limiting Exposure to TimeVarying Electric, Magnetic and Electromagnetic Fields (Up to $300 \mathrm{GHz}$ )," Health Physics, Vol. 74, No. 4, 1998, pp. 494-522.

[3] IEEE, "IEEE Standard for Safety Levels with Respect to Human Exposure to Radio Frequency Electromagnetic Fields, $3 \mathrm{kHz}$ to $300 \mathrm{GHz}$," IEEE STD 95.1, New York, 2005.

[4] J. C. Lin, "Dosimetric Comparison between Different Quantities for Limiting Exposure in the RF Band: Rationale and Implications for Guidelines," Health Physics, Vol. 92, No. 6, 2007, pp. 547-553. doi:10.1097/01.HP.0000236788.33488.65

[5] M. Damm, C. Nusshold, D. Cantillo, G. N. Rechberger, K. Gruber, W. Sattler and C. O. Kappe, "Can Electromagnetic Fields Influence the Structure and Enzymatic Digest of Proteins? A Critical Evaluation of Microwave-Assisted
Proteomics Protocols," Journal of Proteomics, Vol. 75, No. 18, 2012, pp. 5533-5543.

doi:10.1016/j.jprot.2012.07.043

[6] K. R. Foster and R. Glaser, "Thermal Mechanisms of Interaction of Radiofrequency Energy with Biological Systems with Relevance to Exposure Guidelines," Health Physics, Vol. 92, No. 6, 2007, pp. 609-620. doi:10.1097/01.HP.0000262572.64418.38

[7] F. Niedermayr, N. Leitgeb and W. Siegl, "Simulation of the Temperature Elevation in Children Exposed to Plane Wave Electromagnetic Fields (10 MHz - $1 \mathrm{GHz})$ at ICNIRP Reference Level," Biomedical Engineering, Vol. 57, No. 3, 2012, pp. 193-200.

[8] J. F. Bakker, M. M. Paulides, E. Neufeld, A. Christ, N. Kuster and G. C. van Rhoon, "Children and Adults Exposed to Electromagnetic Fields at the ICNIRP Reference Levels: Theoretical Assessment of the Induced Peak Temperature Increase," Physics in Medicine and Biology, Vol. 56, No. 15, 2011, pp. 4967-4989. doi:10.1088/0031-9155/56/15/020

[9] J. F. Bakker, M. M. Paulides, A. Christ, N. Kuster and G. C. van Rhoon, "Assessment of Induced $S A R$ in Children Exposed to Electromagnetic Plane Waves between 10 $\mathrm{MHz}$ and $5.6 \mathrm{GHz}$," Physics in Medicine and Biology, Vol. 55, No. 11, 2010, pp. 3115-3130. doi:10.1088/0031-9155/55/11/009

[10] S. Kuehn, W. Jennings, A. Christ and N. Kuster, "Assessment of Induced Radio-Frequency Electromagnetic Fields in Various Anatomical Human Body Models," Physics in Medicine and Biology, Vol. 54, No. 4, 2009, pp. 875-890. doi:10.1088/0031-9155/54/4/004

[11] E. Conil, A. Hadjem, F. Lacroux, M. F. Wong and J. Wiart, "Variability Analysis of SAR from $20 \mathrm{MHz}$ to 2.4 GHz for Different Adult and Child Models Using Finite Difference Time-Domain," Physics in Medicine and Biology, Vol. 53, No. 6, 2008, pp. 1511-1525. doi:10.1088/0031-9155/53/6/001

[12] P. Dimbylow and W. Bolch, "Whole-Body Average $S A R$ from $50 \mathrm{MHz}$ to $4 \mathrm{GHz}$ in the University of Florida Child Voxel Phantoms," Physics in Medicine and Biology, Vol. 52, No. 22, 2007, pp. 6639-6649. doi:10.1088/0031-9155/52/22/006

[13] J. Q. Wang, O. Fujiwara, S. Kodera and S. Watanabe, "FDTD Calculation of Whole-Body Average $S A R$ in Adult and Child Models for Frequencies from $30 \mathrm{MHz}$ to $3 \mathrm{GHz}$," Physics in Medicine and Biology, Vol. 51, No. 17, 2006, pp. 4119-4127. doi:10.1088/0031-9155/51/17/001

[14] T. Nagoaka, E. Kuneida and S. Watanabe, "ProportionCorrected Scaled Voxel Models for Japanese Children and Their Application to the Numerical Dosimetry of Specific Absorption Rate for Frequencies from $30 \mathrm{MHz}$ to $3 \mathrm{GHz}$," Physics in Medicine and Biology, Vol. 53, No. 23, 2006, pp. 6695-6711 doi:10.1088/0031-9155/53/23/004

[15] P. Bernardi, M. Cavagnaro, S. Pisa and E. Piuzzi, "Specific Absorption Rate and Temperature Elevation in a Subject Exposed in the Far-Field of Radio-Frequency Sources Operating in the $10-900 \mathrm{MHz}$ Range," IEEE 
Transactions on Biomedical Engineering, Vol. 50, No. 3, 2003, pp. 295-304. doi:10.1109/TBME.2003.808809

[16] CST Studio Suite CST GmbH, Bad NauheimerStraße 19, D-64289, Darmstadt, 2009. http://www.cst.com

[17] T. Weiland, "A Method for Discretically Solving Maxwell's Equations for Six-Component Fields (German)," AEÜ, Vol. 31, 1977, pp. 16-120.

[18] A. Christ, W. Kainz, E. G. Hahn, K. Honegger, M. Zefferer, E. Neufeld, W. Rascher, R. Janka, W. Bautz, J. Chen, B. Kiefer, P. Schmitt, H.-P. Hollenbach, J. Shen, M. Oberle, D. Szczerba, A. Kam, J. W. Guag and N. Kuster, "The Virtual Family-Development of Surface-Based Anatomical Models of Two Adults and Two Children for Dosimetric Simulations," Physics in Medicine and Biology, Vol. 55, No. 23, 2010, pp. 23-38. doi:10.1088/0031-9155/55/2/N01

[19] H. Pennes, "Analysis of Tissue and Arterial Blood Temperatures in the Resting Human Foream," Journal of Applied Physiology, Vol. 1, No. 2, 1948, pp. 93-122.

[20] C. Gabriel, S. Gabriel and E. Corthout, "The Dielectric Properties of Biological Tissues: I. Literature Survey," Physics in Medicine and Biology, Vol. 41, No. 11, 1996, pp. 2231-2249. doi:10.1088/0031-9155/41/11/001

[21] S. Gabriel, R. Lau and C. Gabriel, "The Dielectric Properties of Biological Tissues: II. Measurements in the Frequency Range $10 \mathrm{~Hz}$ to $20 \mathrm{GHz}$," Physics in Medicine and Biology, Vol. 41, No. 11, 1996, pp. 2251-2269. doi:10.1088/0031-9155/41/11/002

[22] S. Gabriel, R. Lau and C. Gabriel, "The Dielectric Properties of Biological Tissues: III. Parametric Models for the Dielectric Spectrum of Tissues," Physics in Medicine and Biology, Vol. 41, No. 11, 1996, pp. 2271-2293. doi:10.1088/0031-9155/41/11/003

[23] A. Hirata, H. Sugiyama and M. Kojima, "Computational Model for Calculating Body-Core Temperature Elevation in Rabbits Due to Whole-Body Exposure at $2.45 \mathrm{GHz}$," Physics in Medicine and Biology, Vol. 53, No. 12, 2008, pp. 3391-3404. doi:10.1088/0031-9155/53/12/022
[24] A. Hirata, T. Asano and O. Fujiwara, "FDTD Analysis of Human Body-Core Temperature Elevation Due to RF FarField Energy Prescribed in the ICNIRP Guidelines," Physics in Medicine and Biology, Vol. 52, No. 16, 2007, pp. 5013-5023. doi:10.1088/0031-9155/52/16/020

[25] R. Findlay and P. Dimbylow, "Variations in Calculated $S A R$ with Distance to Perfectly Matched Layer Boundary for a Human Voxel Model," Physics in Medicine and Biology, Vol. 51, No. 23, 2006, pp. 411-415. doi:10.1088/0031-9155/51/23/N02

[26] EN 62311, "Assessment of Electronic and Electrical Equipment Related to Human Exposure Restrictions for Electromagnetic Fields $(0 \mathrm{~Hz}-300 \mathrm{GHz}), " 2008$.

[27] E. Adair, S. A. Kelleher, G. W. Mack and T. S. Morocco, "Thermophysiological Responses of Human Volunteers during Controlled Whole Body Radio Frequency Exposure at $450 \mathrm{MHz}$," Bioelectromagnetics, Vol. 19, No. 4, 1998, pp. 232-245. doi:10.1002/(SICI)1521-186X(1998)19:4<232::AID-BE $\mathrm{M} 5>3.0 . \mathrm{CO} ; 2-2$

[28] F. Niedermayr, "Human Thermoregulation Model of RFEMF Interaction," Ph.D. Thesis, Graz University of Technology, Graz, 2012.

[29] ICNIRP, "ICNIRP Statement on the 'Guidelines for Limiting Exposure to Time-Varying Electric, Magnetic and Electromagnetic Fields (Up to $300 \mathrm{GHz}$ )', Health Physics, Vol. 97, No. 3, 2009, pp. 257-258. doi:10.1097/HP.0b013e3181aff9db

[30] R. L. McIntosh and V. Anderson, "SAR versus VAR, and the Size and Shape That Provide the Most Appropriate RF Exposure Metric in the Range of $0.5-6 \mathrm{GHz}$," Bioelectromagnetics, Vol. 32, No. 4, 2011, pp. 312-321. doi:10.1002/bem.20642

[31] R. L. McIntosh and V. Anderson, "SAR versus S(inc): What Is the Appropriate RF Exposure Metric in the Range 1 - 10 GHz? Part II: Using Complex Human Body Models," Bioelectromagnetics, Vol. 31, No. 6, 2010, pp. 467 478. 